\title{
Structural brain variation, age, and response time
}

\author{
RICHARD J. HAIER \\ University of California, Irvine, California \\ REX E. JUNG and RONALD A. YEO \\ University of New Mexico, Albuquerque, New Mexico \\ and \\ KEVIN HEAD and MICHAEL T. ALKIRE \\ University of California, Irvine, California
}

\begin{abstract}
Response time (RT) generally slows with aging, but the contribution of structural brain changes to this slowing is unknown. We used voxel-based morphometry (VBM) to determine gray matter (GM) and white matter (WM) brain volumes in 9 middle-aged adults (38-58 years old) and 9 seniors (66-82 years old). We correlated brain volumes with RT assessed in both a simple visual stimulus-response task and a visual continuous recognition memory task. No GM correlations with simple RT were significant; there was one WM correlation in the right fusiform gyrus. In the memory task, faster RT was correlated $(p<.05$, corrected) with less GM in the globus pallidus, the parahippocampus, and the thalamus for both groups. Several Brodmann areas (BA) differed between the groups such that in each area, less GM was correlated with slower RTs in the middle-aged group but with faster RTs in the senior group (BAs 19, $37,46,9,8,6,13,10,41$, and 7). The results suggest that individual differences in specific brain structure volumes should be considered as potential moderating factors in cognitive brain imaging studies.
\end{abstract}

Structural brain imaging studies with magnetic resonance imaging (MRI) reveal large individual differences in brain size and morphometry in normal subjects (Allen, Damasio, \& Grabowski, 2002; Good et al., 2001). A number of studies have examined some morphometric differences as they relate to performance on cognitive tasks, but little is known about morphometric differences as they relate to individual differences in speed-of-processing measures such as response time (RT). One study used region-of-interest (ROI) methods and reported that increased volume of the thalamus was correlated with faster performance on speeded neuropsychological tests (measured in seconds) in young and middle-aged adults, but not in older subjects (Van der Werf et al., 2001). A similar ROI study found no age-independent relationships between speeded neuropsychological test performance and volumes of the hippocampus, parahippocampus, or total brain volume (Tisserand, Visser, van Boxtel, \& Jolles, 2000). We are aware of only one study that examined correlations between brain morphometry in several ROIs and RT performance, finding correlations (uncorrected for multiple comparisons) limited to cerebellum measures and variability in RT in a choice RT task (Kamitani, Kuroiwa, Li, Ikegami, \& Matsubara, 2003).

Voxel-based morphometry (VBM) is a newer technique with several advantages over the ROI technique, including automated algorithms to distinguish both gray and white

Correspondence concerning this article should be addressed to R. J. Haier, Med Sci I, B140, ZC5000, University of California, Irvine, CA 92697 (e-mail: rjhaier@uci.edu). matter regional differences across the entire brain (Ashburner \& Friston, 2001; Good et al., 2002; Richardson et al., 1997). VBM was used in one study to determine that the posterior hippocampi in London taxi drivers were larger than those of controls (Maguire et al., 2000). A subsequent study of drivers without taxi experience showed no association between the size of hippocampi and navigation expertise, suggesting that structural differences in the human hippocampus may depend on use rather than reflect innate ability (Maguire et al., 2003). However, a review of 33 studies showing a relationship between hippocampus size and memory noted little evidence to support the "bigger is better hypothesis"; in fact there is evidence that smaller volume predicts better memory for younger subjects (Van Petten, 2004).

Given the paucity of information about the relationship between regional brain structure and RT, we undertook this exploratory VBM study to determine whether regional gray matter (GM) and white matter (WM) volumes are related to speed of processing. Since RT measures generally show age-related slowing and since there are well-known age-related changes in regional brain structures (Tisserand \& Jolles, 2003; Van Petten et al., 2004), we examined both middle-aged and senior adults. Only the thalamus, hippocampus, and parahippocampus have been studied in previous research designed to link neuropsychological test speed to ROI morphometry (Tisserand et al., 2000; Van der Werf et al., 2001); however, use of VBM assesses the entire brain. We hypothesized (1) that RT in a memory task would correlate with GM or WM volumes in areas previously associated with memory 
(Cabeza \& Nyberg, 2000; Tisserand \& Jolles, 2003; Van Petten et al., 2004) and (2) volume in these areas would not be related to RT in a simple stimulus-response task. More GM (or WM) may result in faster RT if more tissue is associated with more resources available to process information for a faster result. Alternatively, more GM (or WM) tissue might also result in slower RT if greater volume is associated with the need for more synapses to fire before a response is made. Given that there are a number of reports of inverse correlations between GM volume and memory performance in older adults (Salat, Kaye, \& Janowsky, 2002; Van Petten et al., 2004) and given that many functional imaging studies show inverse as well as positive correlations between cognitive performance and brain activity (Alkire, Haier, Fallon, \& Cahill, 1998; Daselaar, Prince, \& Cabeza, 2004; Gur et al., 2000; Haier \& Benbow, 1995; Haier et al., 1992; Haier et al., 1988; Landau, Schumacher, Garavan, Druzgal, \& D'Esposito, 2004; Rypma, Berger, \& D’Esposito, 2002; Shaywitz et al., 2001), there is no clear empirical basis for predicting the direction of any correlations between RT and specific regional brain volumes. This would be especially true if any such relationships are age dependent, as are other memory performance/brain structure relationships (Rypma, Prabhakaran, Desmond, \& Gabrieli, 2001; Tisserand \& Jolles, 2003; Van Petten et al., 2004).

\section{METHOD}

All procedures were in accord with the University of California, Irvine, Human Subjects Committee's review and approval; the subjects gave their informed consent. We used a data set from 18 normal right-handed volunteers in good health who had been selected as control subjects for another study (Haier, Alkire, et al., 2003). All subjects with a complete data set including MRI and RT testing were used in this study. There were 10 men and 8 women (mean age $=60.6 ; S D=14.7$; range, $38-82$ ). These subjects were originally selected in two groups: middle-aged $(n=9$, mean age $=47.3$; $S D=6.7$; range, $38-58)$ and seniors $(n=9 ;$ mean age $=73.8$; $S D=4.7$; range, $66-82$ ). Subjects were screened and were included only if they had no medical history of head injury or psychiatric disorder and no indication of dementia or mild cognitive impairment at the time of cognitive testing.

Each subject was tested on two computer-based cognitive tests used previously for a study of neuropharmacological effects on cognition (Polich \& Gloria, 2001). After a tutorial on each test, the subject completed a practice series and then completed the actual experimental trials. The first test was a simple stimulus-response task: After a random delay of 500 to $1,500 \mathrm{msec}$, a cross stimulus was presented in the center of a computer screen for $750 \mathrm{msec}$ or until the subject responded by pressing a button. There were 22 trials. The second test was more difficult: a memory-loaded, continuous recognition test. The stimuli consisted of words, nonsense words, numbers, letters, geometrical shapes, outline drawings of common objects, or human faces from high school year books. During the test, one of these stimuli was presented in the center of the screen and the subject pressed the left arrow key if it had not been presented previously at any time during the foregoing trials and the right arrow key if it had been presented previously. The stimulus was removed with the response. After a 1,000-msec delay, a new stimulus appeared. There were 75 trials; the number of stimuli between two presentations of the same stimulus was random. No stimulus was repeated more than twice, and each category of stimulus had an equal probability of appearing. On average, 37 stimuli sep- arated the presentation of any two identical stimuli. All subjects completed the test in less than $4 \mathrm{~min}$.

Structural MRIs were obtained with a 1.5T clinical Philips Eclipse scanner (Philips Medical Systems, Bothell, WA) on a separate occasion. We used $\mathrm{T}_{1}$-weighted, volumetric spoiled gradient recalled acquisition $(\mathrm{SPGR}) \mathrm{MRI}$ scans $(\mathrm{FOV}=24 \mathrm{~cm}$, flip angle $=40, \mathrm{TR}=24$, $\mathrm{TE}=5$ ). The images consisted of 120 contiguous $1.2-\mathrm{mm}$ thick axial slices, each with an in-plane image matrix of $256 \times 256$ image elements. All images were visually inspected to ensure image quality.

We used voxel-based morphometry (VBM) to identify brain areas where GM and WM volumes were correlated with median RTs in both RT tasks. We used Statistical Parametric Mapping software (SPM2; Wellcome Department of Imaging Neuroscience, University College London) to create a study-specific template and then applied the optimized VBM protocol using the methods of Ashburner and Friston (2000) and Good et al. (2001). To preserve the amount of tissue in any given anatomical region after spatial normalization, the optimal GM, WM, and CSF partitions were multiplied by the Jacobian determinants of their respective spatial transformation matrix. This modulation step was performed so that the final VBM statistics would reflect local deviations in the absolute amount (volume) of tissue in different regions of the brain (Ashburner \& Friston, 2000). The modulated GM and WM partitions were then smoothed with a 12-mm FWHM isotropic Gaussian kernel to account for slight misalignments of homologous anatomical structures and to ensure statistical validity under parametric assumptions.

We used a statistical conjunction approach (Price \& Friston, 1997) to test where both samples had the same or different correlations between RT and GM or WM tissue. There were four basic conjunction analyses (each was repeated separately for each RT task and for GM or WM): (1) areas with a positive correlation between RT and GM in both the middle-aged and the senior groups; (2) areas with a negative correlation in both groups; (3) areas with a negative correlation in the middle-aged group but a positive correlation in the seniors; (4) areas with a positive correlation in the middle-aged, but a negative correlation in the seniors. The first two analyses showed where the groups were the same with respect to any RT and brain structure relationships, and the second two showed where the groups differed. Note that positive correlations show that longer (i.e., slower) RT is associated with more volume. Negative correlations show that shorter (i.e., faster) RT is associated with more volume.

The SPM2 design matrix used with the conjunction analyses to determine GM or WM volume correlations with the simple RT task included sex and total intracranial volume (TIV; the sum of total GM, WM, and CSF volumes) as nuisance covariates. The same design matrix was used for RT in the memory task, except that number of errors was included as an additional nuisance variable as was $\mathrm{RT}$ in the simple task (RT in both tasks was correlated; $r=.58, p<$ $.01)$. Data are presented as significant at $p<.05$, corrected for multiple comparisons (Friston, Worsley, Frackowiak, Mazziotta, \& Evans, 1994; Worsley, Evans, Marrett, \& Neelin, 1992; Worsley et al., 1996). All significant voxel cluster sizes greater than 1 are shown. Coordinates of clusters (centroids) are converted from original Montreal Neurological Institute (MNI) coordinates to those of the Talairach brain atlas (Talairach \& Tournoux, 1988). Anatomical locations of the significant areas are based on the best estimate from the Talairach atlas. The portion of maximum variance accounted for by each correlation (at the centroid) was calculated as $r^{2}=t^{2} /\left(d f+t^{2}\right)$, using $d f=12$ and 10 , respectively, for simple RT and memory RT analyses (from the SPM design matrix).

\section{RESULTS}

In the simple stimulus-response task, the average median RT was $283 \mathrm{msec}(S D=38)$ for the middle-aged group and $307 \mathrm{msec}(S D=63)$ for the seniors. Two subjects made 1 error each, and 16 subjects made no errors. 
In the memory task, the average median RT for the middleaged subjects was $770 \mathrm{msec}(S D=150)$; the mean number of errors was $8.0(S D=5.4)$. For the seniors, the average median RT was $801 \mathrm{msec}(S D=84)$; the mean number of errors was $8.0(S D=2.0)$. Although the seniors tended to be slower in both tasks, RTs did not significantly differ between the two groups.

In the simple stimulus-response task, there were no significant correlations $(p<.05$, corrected for multiple comparisons) between RT and GM in any of the four conjunctions. For WM, there was a single significant finding in the conjunction reflecting a negative correlation in both groups. This was in the right fusiform gyrus, BA 37 (53 voxels, $x=37, y=-49, z=-12, \mathrm{~T}=4.60$ ).

In the continuous recognition memory task, two of the conjunction combinations showed significant GM correlations with $\mathrm{RT}(p<.05$, corrected). In the first conjunction, both the middle-aged and the senior groups showed positive correlations between RT and GM in the right and left globus pallidus, in the left thalamus, and in the left parahippocampus. The locations are detailed in Table 1 and shown in Figure 1A. These are areas where faster RT is associated with less GM volume. In the second conjunction, there were several areas where the middleaged group showed a negative correlation between GM and RT, but in which there was a positive correlation in the seniors. These areas are detailed in Table 2 and shown in Figure 1B. They include BAs 46, 9, 10, and 6 in the left frontal lobe and BAs 8 and 9 in the right medial frontal gyrus; BAs 19 and 37 in the right occipital lobe, BA 13 in the right insula, and BA 41 in the right temporal gyrus. These are areas where faster RTs are associated with more GM in the middle-aged group, but with less GM in the seniors.

There were no significant correlations in the conjunction where both groups had negative correlations between RT and GM or in the conjunction where middleaged subjects showed positive correlations and seniors showed negative ones. There were no significant WM/RT correlations in any of the four conjunction combinations for the memory task.

\section{DISCUSSION}

To our knowledge, this is the first report using VBM to determine whether regional brain structure variation is related to individual differences in RT. Faster RT on a memory task was correlated with less GM in three brain areas - the globus pallidus, the thalamus, and the parahippocampus - in both the middle-aged and the senior groups. Similarly, for the seniors, faster RT was associated with less GM in several Brodmann areas, especially in BAs 46, 9, and other parts of the frontal lobe, whereas less GM in the same areas was associated with slower RT in the middle-aged group. The globus pallidus, thalamus, parahippocampus, and BAs 46/9 have all been reported to be associated with memory (Braver et al., 2001; Cabeza et al., 2004; Gambaryan \& Sarkisyan, 1983; Van der Werf et al., 2003), particularly working memory (Cabeza \& Nyberg, 2000). The inverse relationships in these areas between GM and RT found in this study are consistent with earlier ROI morphometric studies showing inverse correlations between regional brain volume and performance on memory tasks (Salat et al., 2002; Van Petten et al., 2004), although one earlier study found that faster RT was related to a larger thalamus in young and middle-aged adults (Van der Werf et al., 2001).

Currently, it is not known why less tissue is related to faster RT; however, this observation is consistent with a growing body of evidence that regionally efficient brain function and structure may be associated with better cognitive performance (Cabeza et al., 2004; Daselaar et al., 2004; Haier et al., 1995; Haier et al., 1992; Haier et al., 1988; Landau et al., 2004; Salat et al., 2002; Van Petten et al., 2004) and even possible compensatory mechanisms (Cabeza, Anderson, Locantore, \& McIntosh, 2002; Haier, Alkire, et al., 2003). Irrespective of direction, we note that the brain regions identified by VBM to be associated with continuous recognition memory in these samples, including the globus pallidus, thalamus, and BAs $9 / 10$, overlap substantially with the dorsolateral prefrontal circuit (Cummings, 1993) and its afferent connections (BAs 46 and 7). This fronto-subcortical circuit is implicated in mediation of memory storage and retrieval strategies (Tekin \& Cummings, 2002). Thus, VBM may help to elucidate interactions between age and brain circuits largely inaccessible to ROI approaches.

Since there is so little data on RT and brain structure, the apparent interactions among age, GM, and RT should be viewed as interesting, but tentative, empirical observations. Additional hypothesis testing will be necessary, especially in larger samples where VBM becomes more stable (Davatzikos, 2004; Friston \& Ashburner, 2004) and anatomical localization becomes more exact. For ex-

Table 1

Areas Where GM and RT to the Memory Task are Positively Correlated $(p<.05$, Corrected) in Both Middle-Aged and Senior Groups

\begin{tabular}{lccrrrr}
\hline \multicolumn{1}{c}{ Nearest GM Area } & Cluster Size & $x$ & $y$ & \multicolumn{1}{c}{$z$} & T & $r^{2}$ \\
\hline Right medial globus pallidus & 65 & 20 & -12 & -1 & 5.07 & .72 \\
Left parahippocampal gyrus, BA 27 & 584 & -6 & -34 & 0 & 4.93 & .71 \\
Left thalamus & & -6 & -26 & -2 & 4.82 & .70 \\
Left lateral globus pallidus & 3 & -18 & -13 & 0 & 4.41 & .66 \\
\hline
\end{tabular}

Note-GM, gray matter; RT, response time; BA, Brodmann area; $x$-, $y$-, $z$-coordinates are from the Talairach atlas. 


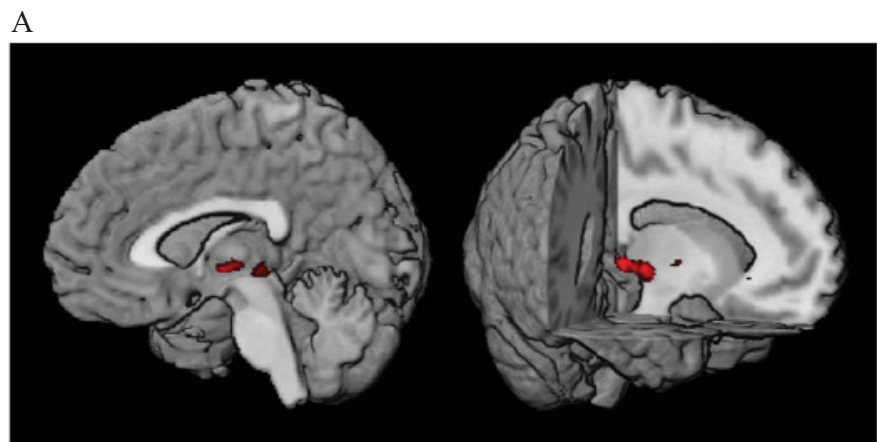

B

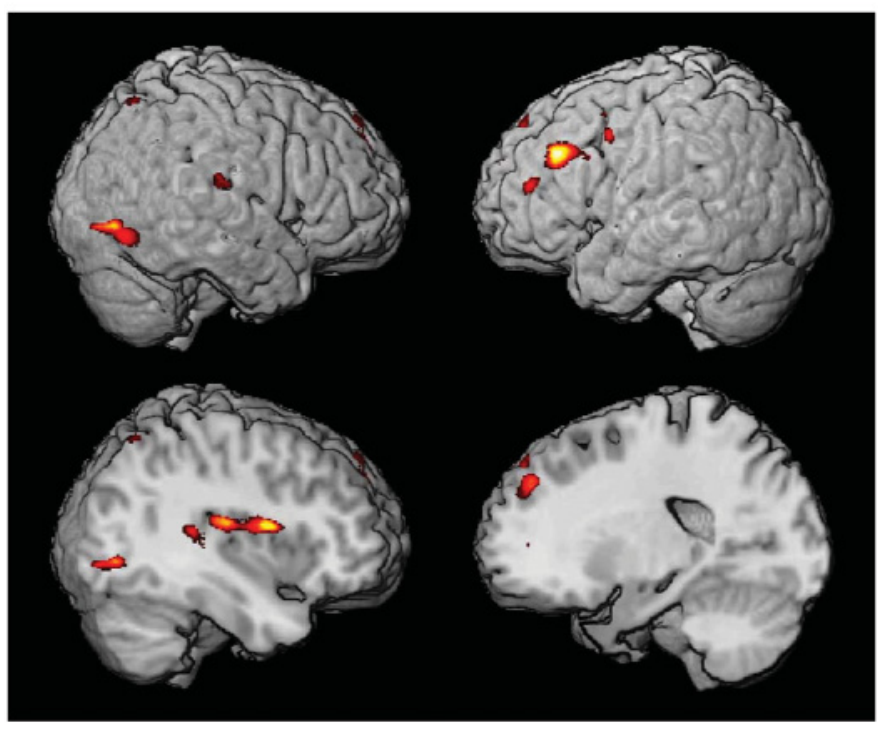

Figure 1. (A) The conjunction of where gray matter and response time (RT) in the continuous recognition memory task are correlated. These are positive correlations common to both samples showing that faster RTs are related to less GM in the left thalamus and to less gray matter in the left parahippocampus (sagittal view in left panel) and in the right globus pallidus (cut-away in right panel). The $x-, y$-, $z$-coordinates of these areas (centroids) are shown in Table 1 ( $p<.05$, corrected for multiple comparisons). (B) Four sagittal views of the conjunction of where RT in the continuous recognition memory task and gray matter is negatively correlated in the middle-aged sample but positively correlated in the seniors. In these areas, faster RT is related to less gray matter in the senior group but to more gray matter in the middle-aged group. The coordinates and names of these areas (centroids) are shown in Table $2(p<$ .05 , corrected). The results in panels $A$ and $B$ are displayed on a studyspecific 3-D template $(p<.10$, corrected); images are oriented so that the right side of each image is the right hemisphere.

ample, in a larger sample, our finding in the thalamus might be better described as in the pulvinar and the finding might extend into the red nucleus. Increased statistical power may also identify additional areas and circuits where brain volume is related to RT.

It should also be noted that our senior group tended to have slower RTs than those for the middle-aged group, as expected, but that these differences were not significant. Our senior group performed each task quite well; the variability of RT was less in the seniors than in the middleaged group. It has been noted (Tisserand \& Jolles, 2003) that elderly subjects may show volume decreases linked to poor cognitive performance only in the presence of neuropathology. More representative samples, matched for performance between age categories, may show different results, as may other cognitive tasks designed to differentiate various aspects of memory functions. In addition to matching subject groups on age and sex, it may also be advisable to match subject groups on general intellectual ability, since individual differences in intelligence are correlated with regional brain activation during passive information processing (Haier, White, \& 
Table 2

Areas Where GM and RT to the Memory Task are Positively Correlated ( $p<.05$, Corrected) in the Senior Group and Inversely Correlated in the Middle-Aged Group

\begin{tabular}{lcrrrrr}
\hline \multicolumn{1}{c}{ Nearest GM Area } & Cluster Size & \multicolumn{1}{c}{$x$} & \multicolumn{1}{c}{$y$} & \multicolumn{1}{c}{$\mathrm{T}$} & $r^{2}$ \\
\hline Right inferior occipital gyrus, BA 19 & 750 & 37 & -70 & 1 & 7.67 & .85 \\
Right inferior occipital gyrus, BA 19 & & 42 & -76 & -1 & 6.27 & .80 \\
Right middle occipital gyrus, BA 37 & & 48 & -66 & -5 & 5.42 & .75 \\
Left middle frontal gyrus, BA 46 & 849 & -50 & 31 & 28 & 7.35 & .84 \\
Right medial frontal gyrus, BA 9 & 1,077 & 3 & 48 & 30 & 6.72 & .82 \\
Right medial frontal gyrus, BA 8 & & 2 & 51 & 39 & 4.93 & .71 \\
Left medial frontal gyrus, BA 6 & & -6 & 44 & 32 & 4.36 & .66 \\
Right insula, BA 13 & 597 & 37 & 6 & 12 & 6.09 & .79 \\
Right insula, BA 13 & & 38 & -5 & 14 & 4.29 & .65 \\
Right insula, BA 13 & 518 & 38 & -17 & 16 & 5.22 & .73 \\
Right insula, BA 13 & & 46 & -19 & 18 & 5.10 & .72 \\
Left middle frontal gyrus, BA 10 & 87 & -28 & 45 & 11 & 5.15 & .73 \\
Left middle frontal gyrus, BA 9 & 55 & -42 & 8 & 37 & 4.96 & .71 \\
Right transverse temporal gyrus, BA 41 & 87 & 38 & -33 & 12 & 4.96 & .71 \\
Right superior parietal lobule, BA 7 & 12 & 39 & -59 & 56 & 4.63 & .68 \\
\hline
\end{tabular}

Note-GM, gray matter; RT, response time; BA, Brodmann area; $x$-, $y$-, $z$-coordinates are from the Talairach atlas.

Alkire, 2003) and with regional GM and WM variation (Haier, Jung, Yeo, Head, \& Alkire, 2004, 2005).

Although GM and WM are highly hereditable in some areas more than others (Posthuma et al., 2002; Thompson et al., 2001), there is also some evidence that GM volume increases with practice (Draganski et al., 2004). Whether regional brain volumes influence RT or whether RT experience influences brain volumes, individual differences in total or regional brain volumes appear to be potentially confounding variables to be taken into account in cognitive brain imaging experiments, along with age, sex, and handedness. For example, trained athletes, musicians, or avid computer game players may yield experimental results under some circumstances that do not generalize well to the wider population if structural brain variation is not taken into account. Any effects of structural brain variation are often ignored when whole brain size and shape are normalized in imaging studies that rely on fitting all brains into a standard space for statistical analyses.

Our findings indicate that regional variation in GM volume is related to individual differences in speed of processing in a continuous recognition memory task. This suggests that regional brain volumes should be studied as potential sources of variance to help ascertain functional and structural brain effects independently of one another in functional cognitive imaging experiments. This differentiation is an important step in establishing the relationships among brain structure, function, and cognition.

\section{REFERENCES}

Alkire, M. T., Haier, R. J., Fallon, J. H., \& Cahill, L. (1998). Hippocampal, but not amygdala, activity at encoding correlates with long-term, free recall of nonemotional information. Proceedings of the National Academy of Sciences, 95, 14506-14510.

Allen, J. S., Damasio, H., \& Grabowski, T. J. (2002). Normal neuroanatomical variation in the human brain: An MRI-volumetric study. American Journal of Physical Anthropology, 118, 341-358.
AshbuRner, J., \& FrISTON, K. J. (2000). Voxel-based morphometrythe methods. NeuroImage, 11, 805-821.

Ashburner, J., \& Friston, K. J. (2001). Why voxel-based morphometry should be used. NeuroImage, 14, 1238-1243.

Braver, T. S., Barch, D. M., Kelley, W. M., Buckner, R. L., Cohen, N. J., Miezin, F. M., Snyder, A. Z., Ollinger, J. M., Akbudak, E., Conturo, T. E., \& Petersen, S. E. (2001). Direct comparison of prefrontal cortex regions engaged by working and long-term memory tasks. NeuroImage, 14, 48-59.

Cabeza, R., Anderson, N. D., Locantore, J. K., \& McIntosh, A. R. (2002). Aging gracefully: Compensatory brain activity in highperforming older adults. NeuroImage, 17, 1394-1402.

Cabeza, R., Daselaar, S. M., Dolcos, F., Prince, S., Budde, M., \& NYBERG, L. (2004). Task-independent and task-specific age effects on brain activity during working memory, visual attention and episodic retrieval. Cerebral Cortex, 14, 364-375.

CABEZA, R., \& Nyberg, L. (2000). Imaging cognition II: An empirical review of 275 PET and fMRI studies. Journal of Cognitive Neuroscience, 12, 1-47.

Cummings, J. L. (1993). Frontal-subcortical circuits and human behavior. Archives of Neurology, 50, 873-880.

Daselaar, S. M., Prince, S. E., \& Cabeza, R. (2004). When less means more: Deactivations during encoding that predict subsequent memory. NeuroImage, 23, 921-927.

DavatziKos, C. (2004). Why voxel-based morphometric analysis should be used with great caution when characterizing group differences. NeuroImage, 23, 17-20.

Draganski, B., Gaser, C., Busch, V., Schuierer, G., Bodgahn, U., \& MAY, A. (2004). Neuroplasticity: Changes in grey matter induced by training. Nature, 427, 311-312.

Friston, K. [J.], \& AsHBURNER, J. (2004). Generative and recognition models for neuroanatomy. NeuroImage, 23, 21-24.

Friston, K. J., Worsley, K. J., Frackowiak, R. S. J., Mazziotta, J. C., \& Evans, A. C. (1994). Assessing the significance of focal activations using their spatial extent. Human Brain Mapping, 1, 214220.

Gambaryan, L. S., \& Sarkisyan, Z. S. (1983). Role of the globus pallidus in the mechanisms of memory. Neuroscience \& Behavioral Physiology, 13, 470-475.

Good, C. D., Johnsrude, I. S., Ashburner, J., Henson, R. N. A., FrisTON, K. J., \& FraCKOWIAK, R. S. J. (2001). A voxel-based morphometric study of ageing in 465 normal adult human brains. NeuroImage, 14, 21-36.

Good, C. D., Scahill, R. I., Fox, N. C., Ashburner, J., Friston, K. J., Chan, D., Crum, W. R., Rossor, M. N., \& Frackowiak, R. S. J. (2002). Automatic differentiation of anatomical patterns in the human 
brain: Validation with studies of degenerative dementias. NeuroImage, 17, 29-46.

Gur, R. C., Alsop, D., Glahn, D., Petty, R., Swanson, C. L., MaldJian, J. A., Turetsky, B. I., Detre, J. A., Gee, J., \& Gur, R. E. (2000). An fMRI study of sex differences in regional activation to a verbal and a spatial task. Brain \& Language, 74, 157-170.

Haier, R. J., Alkire, M. T., White, N. S., UnCAPher, M. R., Head, E. LotT, I. T., \& CotTman, C. W. (2003). Temporal cortex hypermetabolism in Down syndrome prior to the onset of dementia. Neurology, 61, 1673-1679.

HAIER, R. J., \& BENBOw, C. P. (1995). Sex differences and lateralization in temporal lobe glucose metabolism during mathematical reasoning. Developmental Neuropsychology, 11, 405-414.

Haier, R. J., Chueh, D., Touchette, P., Lott, I., Buchsbaum, M. S., Macmillan, D., Sandman, C., LaCasse, L., \& Sosa, E. (1995). Brain size and cerebral glucose metabolic rate in nonspecific mental retardation and Down syndrome. Intelligence, 20, 191-210.

Haier, R. J., Jung, R. E., Yeo, R. A., Head, K., \& Alkire, M. T. (2004). Structural brain variation and general intelligence. NeuroImage, 23, 425-433.

Haier, R. J., Jung, R. E., Yeo, R. A., Head, K., \& Alkire, M. T. (2005). The neuroanatomy of general intelligence: Sex matters. NeuroImage, 25, 320-327.

Haier, R. J., Siegel, B. V., Jr., MacLachlan, A., Soderling, E., LotTENBERG, S., \& BuchsBaum, M. S. (1992). Regional glucose metabolic changes after learning a complex visuospatial/motor task: A positron emission tomographic study. Brain Research, 570, 134-143.

Haier, R. J., Siegel, B. V., JR., Nuechterlein, K. H., Hazlett, E., Wu, J. C., Paek, J., Browning, H. L., \& Buchsbaum, M. S. (1988). Cortical glucose metabolic rate correlates of abpstract reasoning and attention studied with positron emission tomography. Intelligence, 12, 199217.

Haier, R. J., White, N. S., \& AlkiRe, M. T. (2003). Individual differences in general intelligence correlate with brain function during nonreasoning tasks. Intelligence, 31, 429-441.

Kamitani, T., Kuroiwa, Y., Li, M., Ikegami, T., \& Matsubara, S. (2003). Relationship between cerebellar size and variation of reaction time during a visual cognitive task in normal subjects. Journal of Neurology, 250, 1001-1003.

Landau, S. M., Schumacher, E. H., Garavan, H., Druzgal, J., \& D'Esposito, M. (2004). A functional MRI study of the influence of practice on component processes of working memory. NeuroImage, 22, 211-221.

Maguire, E. A., Gadian, D. G., Johnsrude, I. S., Good, C. D., AshBURNER, J., Frackowiak, R. S. J., \& Frith, C. D. (2000). Navigationrelated structural change in the hippocampi of taxi drivers. Proceedings of the National Academy of Sciences, 97, 4398-4403.

Maguire, E. A., Siters, H. J., Good, C. D., Hartley, T., Frackowiak, R. S. J., \& Burgess, N. (2003). Navigation expertise and the human hippocampus: A structural brain imaging analysis. Hippocampus, $\mathbf{1 3}$, 250-259.

Polich, J., \& Gloria, R. (2001). Cognitive effects of Ginkgo biloba/ vinpocetine compound in normal adults: Systematic assessment of perception, attention, and memory. Human Psychopharmacology: Clinical \& Experimental, 16, 409-416.

Posthuma, D., De Geus, E. J. C., Baaré, W. F. C., Pol, H. E. H., Kahn, R. S., \& Boomsma, D. I. (2002). The association between brain volume and intelligence is of genetic origin. Nature Neuroscience, $\mathbf{5}, 83-84$.

PrICE, C. J., \& FrISTON, K. J. (1997). Cognitive conjunction: A new approach to brain activation experiments. NeuroImage, 5, 261-270

Richardson, M. P., Friston, K. J., Sisodiya, S. M., Koepp, M. J., AshBURner, J., Free, S. L., Brooks, D. J., \& DUnCAN, J. S. (1997). Cortical grey matter and benzodiazepine receptors in malformations of cortical development: A voxel-based comparison of structural and functional imaging data. Brain, 120, 1961-1973.

Rypma, B., Berger, J. S., \& D'Esposito, M. (2002). The influence of working-memory demand and subject performance on prefrontal cortical activity. Journal of Cognitive Neuroscience, 14, 721-731.

Rypma, B., Prabhakaran, V., Desmond, J. E., \& Gabrieli, J. D. E. (2001). Age differences in prefrontal cortical activity in working memory. Psychology \& Aging, 16, 371-384.

Salat, D. H., Kaye, J. A., \& Janowsky, J. S. (2002). Greater orbital prefrontal volume selectively predicts worse working memory performance in older adults. Cerebral Cortex, 12, 494-505.

Shaywitz, B. A., Shaywitz, S. E., Pugh, K. R., Fulbright, R. K., Skudlarski, P., Mencl, W. E., Constable, R. T., Marchione, K. E., Fletcher, J. M., Klorman, R., Lacadie, C., \& Gore, J. C. (2001). The functional neural architecture of components of attention in language-processing tasks. NeuroImage, 13, 601-612.

TAlairach, J., \& TournOux, P. (1988). Co-planar stereotaxic atlas of the human brain: 3-Dimensional proportional system. An approach to cerebral imaging. New York: Thieme.

Tekin, S., \& Cummings, J. L. (2002). Frontal-subcortical neuronal circuits and clinical neuropsychiatry: An update. Journal of Psychosomatic Research, 53, 647-654

Thompson, P. M., Cannon, T. D., Narr, K. L., van ErP, T., Poutanen, V.-P., HutTUnEN, M., LÖNNQVIST, J., STANDERTSKJÖLD-NORDENSTAM, C.-G., Kaprio, J., Khaledy, M., Dail, R., Zoumalan, C. I., \& Toga, A. W. (2001). Genetic influences on brain structure. Nature Neuroscience, 4, 1253-1258.

Tisserand, D. J., \& Jolles, J. (2003). On the involvement of prefrontal networks in cognitive ageing. Cortex, 39, 1107-1128.

Tisserand, D. J., Visser, P. J., van Boxtel, M. P. J., \& Jolles, J. (2000). The relation between global and limbic brain volumes on MRI and cognitive performance in healthy individuals across the age range. Neurobiology of Aging, 21, 569-576.

VAn Der Werf, Y. D., Scheltens, P., Lindeboom, J., Witter, M. P., Uylings, H. B. [M.], \& Jolles, J. (2003). Deficits of memory, executive functioning and attention following infarction in the thalamus: A study of 22 cases with localised lesions. Neuropsychologia, 41, $1330-1344$

Van der Werf, Y. D., Tisserand, D. J., Visser, P. J., Hofman, P. A. M., Vuurman, E., Uylings, H. B. M., \& Jolles, J. (2001). Thalamic volume predicts performance on tests of cognitive speed and decreases in healthy aging: A magnetic resonance imaging-based volumetric analysis. Cognitive Brain Research, 11, 377-385.

Van Petten, C. (2004). Relationship between hippocampal volume and memory ability in healthy individuals across the lifespan: Review and meta-analysis. Neuropsychologia, 42, 1394-1413.

Van Petten, C., Plante, E., Davidson, P. S. R., Kuo, T. Y., BajusCAK, L., \& GLISKY, E. L. (2004). Memory and executive function in older adults: Relationships with temporal and prefrontal gray matter volumes and white matter hyperintensities. Neuropsychologia, $\mathbf{4 2}$, $1313-1335$

Worsley, K. J., Evans, A. C., Marrett, S., \& Neelin, P. (1992). A three-dimensional statistical analysis for $\mathrm{CBF}$ activation studies in human brain. Journal of Cerebral Blood Flow \& Metabolism, 12, 900-918.

Worsley, K. J., Marrett, S., Neelin, P., Vandal, A. C., Friston, K. J., \& Evans, A. C. (1996). A unified statistical approach for determining significant signals in images of cerebral activation. Human Brain Mapping, 4, 58-73.

(Manuscript received September 15, 2004; revision accepted for publication February 24, 2005.) 